\title{
Observation of Anisotropic Exchange in a Spin Ladder by ESR
}

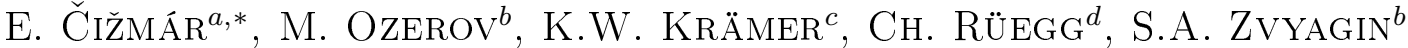 \\ ${ }^{a}$ Institute of Physics, Faculty of Sciences, P.J. Šafárik University, Park Angelinum 9, Košice, Slovakia \\ ${ }^{b}$ Dresden High Magnetic Field Laboratory (HLD), Helmholtz-Zentrum Dresden-Rossendorf, Dresden, Germany \\ ${ }^{c}$ Department of Chemistry and Biochemistry, University of Bern, Bern, Switzerland \\ ${ }^{d}$ Laboratory for Neutron Scattering, Paul Scherrer Institut, Villigen PSI, Switzerland
}

\begin{abstract}
We report on high resolution X-band electron spin resonance (ESR) spectroscopy studies of the spinladder material $\left(\mathrm{C}_{5} \mathrm{H}_{12} \mathrm{~N}\right)_{2} \mathrm{CuBr}_{4}$. Our experiments provide a direct evidence for the presence of anisotropy in $\left(\mathrm{C}_{5} \mathrm{H}_{12} \mathrm{~N}\right)_{2} \mathrm{CuBr}_{4}$ in contrast to a fully isotropic spin-ladder model employed for this system previously. Lowtemperature angular dependence of ESR transitions is analyzed employing a simple spin- $1 / 2$ dimer model with the symmetric anisotropic exchange interaction.
\end{abstract}

DOI: 10.12693 /APhysPolA.126.238

PACS: 76.30.-v, 74.25.Ha, 75.50.Gg, 75.30.Gw

\section{Introduction}

Due to a rich variety of their unusual magnetic properties, quantum spin ladders continue to attract a lot of attention. The material $\left(\mathrm{C}_{5} \mathrm{H}_{12} \mathrm{~N}\right)_{2} \mathrm{CuBr}_{4}$, abbreviated as BPCB, is known as a prototypical realization of the two-leg spin-1/2 antiferromagnetic ladder system in the strong-coupling limit $\left(J_{\text {leg }}<J_{\text {rung }}\right.$, where $J_{\text {leg }}$ and $J_{\text {rung }}$ are leg and rung exchange couplings, respectively) [1]. In zero magnetic field the ground state of BPCB is gapped and quantum disordered. The gapless Luttinger-Liquid phase was observed in BPCB between critical fields $6.8 \mathrm{~T}$ and $13.8 \mathrm{~T}[2,3]$. In addition, the transition into the field-induced magnetically ordered phase occurs below $110 \mathrm{mK}$. The latter can be effectively described in terms of the Bose-Einstein condensation of magnons $[2,4]$.

Here, we report on X-band ESR studies of BPCB. A distinct effect of the anisotropy was revealed at low temperatures. Using a simplified model with anisotropic interaction along rungs, the ESR excitation spectrum is described.

\section{Experiment and discussion}

ESR experiments were performed at the Dresden High Magnetic Field Laboratory (HLD), employing the Xband spectrometer Bruker ELEXSYS E500 at a fixed frequency of $9.4 \mathrm{GHz}$. High-quality single crystals of the deuterated analog of BPCB were used in our experiments. The unit cell of BPCB (space group $P 2_{1} / c$ ) contains two crystallographically equivalent spin-1/2 ladders running along the $a$ axis. The two sets of ladders are characterized by different orientations of the principal axis of the $g$ tensors, which is consistent with previous high-temperature observations [5]. However, a much

*corresponding author; e-mail: erik.cizmar@upjs.sk more complex ESR spectrum was observed below $7 \mathrm{~K}$. In Fig. 1 we present the angular dependence of ESR fields obtained at $3.3 \mathrm{~K}$. The observed angular dependence is incompatible with the simple isotropic spin-ladder model, providing a clear signature of additional anisotropic interactions in BPCB. It is important to mention that the observed behaviour is similar to that obtained for hydrogenated samples. As follows from their temperature dependence, the ESR spectra correspond to transitions within the thermally populated excited triplets. We applied a simplified spin-triplet model with axial and in-plane anisotropy terms $\left(D / k_{B}=0.55 \mathrm{~K}\right.$, and $E / k_{B}=0.05 \mathrm{~K}$, respectively) for the description of $\mathrm{X}$ band and high-frequency ESR data previously [6]. It is important to mention that the effect of anisotropy was observed only at low temperatures (when the effect of thermal fluctuations is absent) and disappeared with increasing temperature. Due to the thermally activated exchange-narrowing effect [7] the low-temperature ESR lines from each ladder merge into a single one above $7 \mathrm{~K}$.

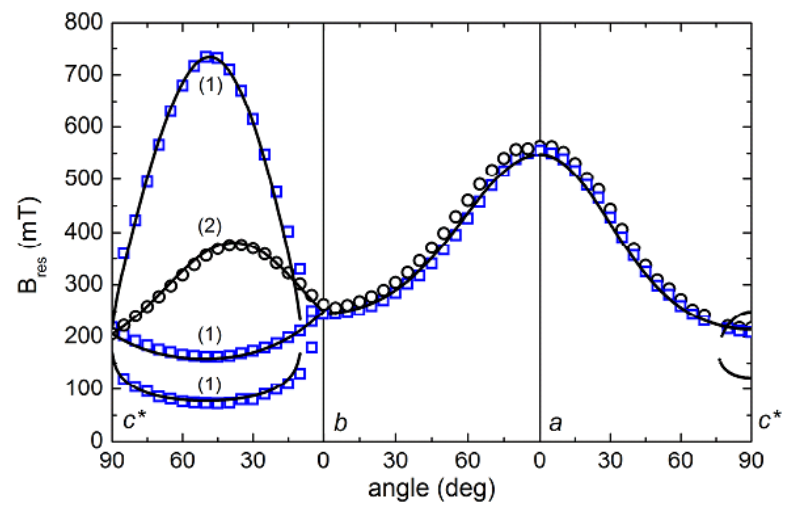

Fig. 1. The angular dependence of ESR resonance fields (symbols) measured at $T=3.3 \mathrm{~K}$ and results of the simulation as described in the text (lines). Labels (1) and (2) denote the two ladders with different orientation of the principal axis of the $g$ tensors. 
An antisymmetric Dzyaloshinskii-Moriya (DM) interaction and/or a symmetric anisotropic exchange (SAE) interaction caused by spin-orbit coupling can be proposed as the main source of the observed anisotropy. By the inversion symmetry, the DM interaction is forbidden on the rungs of the ladder, but it is allowed along the ladder legs. As shown in Ref. [8], the high-frequency ESR measurements [6] can be explained by taking into account the SAE on the ladder rungs and legs. Based on these results, we use a spin-1/2 dimer model (with spins in dimers coupled by the exchange interaction including SAE) given by Hamiltonian

$$
\mathcal{H}=J \hat{S}_{1} \cdot \hat{S}_{2}+\hat{S}_{1} \cdot \bar{\Gamma} \cdot \hat{S}_{2},
$$

where $J$ is the isotropic exchange interaction, $\bar{\Gamma}$ is the tensor of the SAE interaction, and $\hat{S}_{1}$ and $\hat{S}_{2}$ are spin operators $[9,10]$. The anisotropy parameters $\Gamma_{z z}$ and $\left(\Gamma_{x x}-\Gamma_{y y}\right)$, taken from the diagonal components of the SAE tensor play the same role as the parameters $D$ and $E$ [10] used for the ESR simulation in Ref. [6]. The ESR angular dependence was analyzed employing Eq. 1 and the EasySpin simulation package [11]. Results of the simulations are presented in Fig. 1 by solid lines. Good agreement with experimental data was obtained using $g_{x}=$ $2.065, g_{y}=2.045, g_{z}=2.29$ and $\Gamma_{x x} / k_{B}=-0.28 \mathrm{~K}$, $\Gamma_{y y} / k_{B}=-0.48 \mathrm{~K}$ and $\Gamma_{z z} / k_{B}=0.76 \mathrm{~K}$ (resulting in $\left.\Gamma_{x x}-\Gamma_{y y}=0.2 \mathrm{~K}\right)$. In our simulations, an isotropic exchange interaction on the ladder rungs $J / k_{B}=12.9 \mathrm{~K}$ was used [2-4]. The local anisotropy axis of the SAE tensor was found to be tilted counterclockwise by $50^{\circ}$ from the $b$ axis in the $b c^{*}$ plane $\left(c^{*} \perp a, b\right)$ for ladder (1) and clockwise for ladder (2), as labelled in Fig. 1. A tiny splitting of the ESR line was found when rotating the crystal around the $b$ or $c^{*}$ axis. The splitting can be explained by a small (up to $2^{\circ}$ ) misalignment between the experimental axis of rotation and the direction of $b$ or $c^{*}$, respectively. In Fig. 2 we present spectra measured with the magnetic field applied along the local anisotropy axis of the ladder (1). The simulated relative intensities of the resonance lines agree well with the experimental observations.

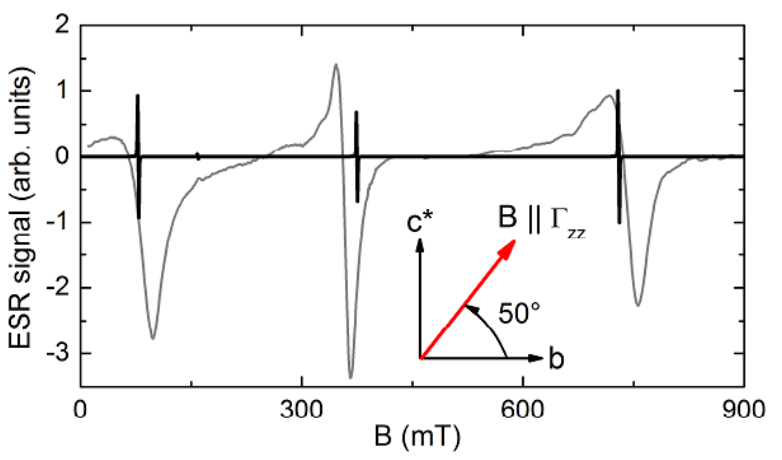

Fig. 2. ESR spectrum (shown in grey) for magnetic field applied along the anisotropy axis of ladder (1) and simulation (shown in black).

\section{Conclusions}

The anisotropy of the magnetic interactions observed in BPCB is an important parameter to be taken into account when describing magnetic properties of spin ladders close to their quantum critical points. Using a simple dimer model (with SAE on the rungs), the angular dependence of the ESR absorption was described and ESR spectra in BPCB were simulated. We have demonstrated that the used approach is a very powerful tool for detecting the subtle anisotropy effects.

\section{Acknowledgments}

This work was supported by EuroMagNET (EU contract No. 228043), VEGA 1/0145/13, APVV-0132-11 and ERDF EU via contract No. ITMS26220120047.

\section{References}

[1] B.C. Watson, V.N. Kotov, M.W. Meisel, D.W. Hall, G.E. Granroth, W.T. Montfrooij, S.E. Nagler, D.A. Jensen, R. Backov, M.A. Petruska, G.E. Fanucci, D.R. Talham, Phys. Rev. Lett. 86 , 5168 (2001).

[2] M. Klanjšek, H. Mayaffre, C. Berthier, M. Horvatić, B. Chiari, O. Piovesana, P. Bouillot, C. Kollath, E. Orignac, R. Citro, T. Giamarchi, Phys. Rev. Lett. 101, 137207 (2008).

[3] Ch. Rüegg, K. Kiefer, B. Thielemann, D.F. McMorrow, V. Zapf, B. Normand, M.B. Zvonarev, P. Bouillot, C. Kollath, T. Giamarchi, S. Capponi, D. Poilblanc, D. Biner, K.W. Krämer, Phys. Rev. Lett. 101, 247202 (2008).

[4] B. Thielemann, Ch. Rüegg, K. Kiefer, H.M. Rønnow, B. Normand, P. Bouillot, C. Kollath, E. Orignac, R. Citro, T. Giamarchi, A.M. Läuchli, D. Biner, K. Krämer, F. Wolff-Fabris, V. Zapf, M. Jaime, J. Stahn, N.B. Christensen, B. Grenier, D.F. McMorrow, J. Mesot, Phys. Rev. B 79, 020408(R) (2009).

[5] B.R. Patyal, B.L. Scott, R.D. Willett, Phys. Rev. B 41, 1657 (1990).

[6] E. Čižmár, M. Ozerov, J. Wosnitza, B. Thielemann, K.W. Krämer, Ch. Rüegg, O. Piovesana, M. Klanjšek, M. Horvatić, C. Berthier, S.A. Zvyagin, Phys. Rev. B 82, 054431 (2010).

[7] P.W. Anderson, J. Phys. Soc. Jpn. 9, 316 (1954).

[8] S.C. Furuya, P. Bouillot, C. Kollath, M. Oshikawa, T. Giamarchi, Phys. Rev. Lett. 108, 037204 (2012).

[9] M.-H. Whangbo, H.-J. Koo, D. Dai, J. Solid State Chem. 176, 417 (2003).

[10] L.M.B. Napolitano, O.R. Nascimento, S. Cabaleiro, J. Castro, R. Calvo, Phys. Rev. B 77, 214423 (2008).

[11] S. Stoll, A. Schweiger, J. Magn. Reson. 178, 42 (2006). 